\title{
Origine de la minéralisation des eaux des aquifères discontinus sous couvert forestier de la zone Sud de la Côte d'Ivoire : cas de la région d'Abidjan-Agboville
}

\author{
E. Kouassi AHOUSSI ${ }^{*}$, Nagnin SORO, B.Yao KOFFI, Gbombélé SORO et \\ Jean BIEMI
}

Laboratoire des Sciences et Techniques de l'Eau et de l'Environnement (LSTEE), UFR des Sciences de la Terre et des Ressources Minières (STRM), Université de Cocody 22 BP 582 Abidjan 22 (Côte d'Ivoire)

*Auteur correspondant, E-mail : ahoussi@gmx.fr / akouassiernest@yahoo.fr

\section{RESUME}

Les eaux souterraines constituent l'une des principales sources d'approvisionnement en eau potable de la population de la région d'Agboville-Abidjan. Cette étude se propose d'améliorer les connaissances sur les nappes souterraines et de déterminer l'origine de la minéralisation des eaux souterraines de la région à partir d'une combinaison des méthodes hydrochimiques et de l'analyse statistique multivariée. L'étude hydrochimique montre que les eaux de la région ont une température moyenne de $27,7 \pm 0,1{ }^{\circ} \mathrm{C}$. Elles sont acides, avec un $\mathrm{pH}$ qui varie entre 3,8 et 6,9. Ces eaux sont faiblement minéralisées, ce qui confirme un trait chimique bien connu dans le pays et une diversité de faciès chimiques en rapport avec la nature lithologique des terrains. Cependant, d'importantes valeurs de la conductivité ont été révélées dans les eaux de certaines localités telles que Gori M'Po $\left(819 \mu \mathrm{S} . \mathrm{cm}^{-1}\right)$ et Lobo Akoudzin $\left(503 \mu \mathrm{S} . \mathrm{cm}^{-1}\right)$. Dans la région, les eaux souterraines se regroupent en quatre hydrofaciès qui sont dominés par les eaux bicarbonatées calciques (61\%) et les eaux chlorurées sodi-potassiques (22\%). Les faciès bicarbonatés sodi-potassiques (10\%), et chlorurés calciques (7\%) sont peu représentés dans ces eaux. Les principaux ions à l'origine de la minéralisation des eaux sont issus de l'altération des roches, de l'hydrolyse des minéraux silicatés tels que l'anorthite dans les plagioclases et de la décomposition des minéraux ferro-magnésiens comme la biotite et l'amphibole présentes dans les roches. A cela s'ajoute le pluviolessivage des sols et une pollution d'origine humaine liée aux activités anthropiques.

(C) 2010 International Formulae Group. All rights reserved.

Mots clés. Abidjan-Agboville, analyse multivariée, eaux souterraines et hydrochimie.

\section{INTRODUCTION}

En Côte d'Ivoire, l'accès à l'eau potable des populations constitue l'une des préoccupations majeures pour l'Etat ivoirien. C'est pourquoi, depuis son indépendance, le pays s'est doté d'infrastructures hydrauliques adéquates pour garantir une alimentation en eau potable de sa population. Cependant, face à une croissance démographique importante, les besoins en eau des populations se sont de plus en plus accrus et la majeure partie des ouvrages réalisés sont devenus insuffisants, ce qui entraîne des pénuries d'eau dans le pays. La zone d'Abidjan-Agboville, à l'instar de toutes les autres localités ivoiriennes, est également confrontée à ces problèmes d'eau 
qui sont récurrents dans la zone de socle, sous couvert forestier. Cette région, d'environ 5 millions d'habitants (INS, 2001), est confrontée à une énorme croissance démographique, liée à l'exode massif des populations venant des autres régions à la recherche de fortune. Dans cette zone, les nappes de fractures sont peu productives. Les débits rencontrés lors des forations sont faibles et varient entre $0,5 \mathrm{~m}^{3} / \mathrm{h}$ et $17 \mathrm{~m}^{3} / \mathrm{h}$ (Ahoussi, 2008) pour des grandes agglomérations comme la ville d'Agboville. Aussi, face à cette insuffisance des eaux souterraines, la Société de Distribution d'Eau de Côte d'Ivoire (SODECI), s'est-elle tournée vers les eaux de surface par le captage de l'eau de la rivière Agbô pour l'alimentation en eau potable des populations de la ville d'Agboville. Depuis ces dix dernières années, la qualité de l'eau de cette rivière soumise à l'eutrophisation s'est considérablement dégradée au point que les traitements ordinaires n'arrivent plus à purifier l'eau (N'go et al., 2005). La qualité de l'eau de robinet cause régulièrement des désagréments aux populations de la ville d'Agboville qui se tournent de plus en plus vers les eaux des aquifères discontinus de la région. Aussi, dans les villages les eaux de ces aquifères sont-elles couramment captées par de nombreux ouvrages hydrauliques et constituent la seule alternative de production d'eau potable. Ces nappes d'eaux souterraines sont mal connues. Ainsi la présente étude se propose d'étudier l'origine des différents processus qui gouvernent la minéralisation de ces eaux souterraines en zone forestière.

\section{Principaux traits de la zone d'étude}

Le secteur d'étude est situé au Sud de la Côte d'Ivoire en zone forestière entre les latitudes $5^{\circ} 30$ et $6^{\circ} 00$ Nord et les longitudes $3^{\circ} 30$ et $4^{\circ} 20$ Ouest (Figure 1 ). Le climat de la région est de type équatorial dans la zone littorale, et tropical humide vers Agboville. Cette région est une zone pluvieuse, avec des précipitations interannuelles supérieures à $1500 \mathrm{~mm}$. Elle est caractérisée par quatre saisons dont deux saisons pluvieuses, une grande saison de pluie d'avril à juillet et une petite saison de pluie d'octobre à novembre. Ces deux saisons sont entrecoupées par deux périodes sèches qui s'étendent de décembre à mars et d'août à septembre. La végétation évolue de la forêt claire sur le littoral au Sud à la forêt dense sempervirente et ombrophile vers le Nord de la région. Cette forêt est largement dégradée suite à la croissance de l'habitat humain, au développement de l'agriculture et à l'exploitation abusive de la forêt qui l'ont réduite à de petits îlots dont certains ont été classés. La région renferme également un réseau hydrographique très dense composé du fleuve Comoé et des rivières Mé et Agnéby ainsi que de leurs affluents.

$\mathrm{Du}$ point de vue géologique, la région d'Abidjan-Agboville appartient à un ensemble formé de deux unités géologiques dont le bassin sédimentaire situé au Sud et le socle cristallin et cristallophyllien au Nord. Les formations du socle qui affleurent dans la région sont attribuées au Paléoprotérozoïque et définissent le cycle de dépôt birimien (Delor et al., 1992). Leur origine est essentiellement sédimentaire, avec un fort pourcentage de faciès détritiques grossiers. Le socle cristallin est constitué de formations birimiennes qui sont les schistes, les métaarénites et les métasiltstones. Dans ces formations, apparaissent en intrusions des granitoïdes éburnéens composés de gneiss, de granites, de granito-gneiss, auxquels sont associés des auréoles de métamorphisme. L'altération de ces formations produit une épaisse couche d'altérites dont la puissance varie entre 4 et $40 \mathrm{~m}$ (N'go et al., 2005). Le socle est de manière générale incliné du Nord au Sud en direction de l'Océan Atlantique, avec une pente qui varie entre 6 et $9 \%$.

\section{MATERIEL ET METHODES}

Les analyses complètes ont été réalisées sur les eaux de forage captant les nappes de fractures de la région d'AbidjanAgboville. Ces analyses chimiques couvrent toutes la zone de socle et visent à déterminer 
les caractéristiques chimiques des eaux. Cette étude a été effectuée sur 42 échantillons d'eau prélevés dans le socle cristallin (Figure 2) au niveau des forages et des puits. Sur ces eaux, les paramètres physiques tels que la température, le $\mathrm{pH}$, la conductivité électrique (CE) de l'eau ont été mesurés in situ à l'aide d'un pH-mètre et d'un conductimètre de marque WTW 330. Les échantillons d'eau destinés aux analyses chimiques ont été recueillis dans des bouteilles en polyéthylène et transportés au Laboratoire d'Analyse d'Abengourou (LPA) pour être analysés. Les paramètres chimiques analysés sont les cations : $\mathrm{Ca}^{2+}, \mathrm{Mg}^{2+}, \mathrm{K}^{+}$et $\mathrm{Na}^{+}$; les anions : $\mathrm{SO}_{4}{ }^{2-}, \mathrm{Cl}^{-}, \mathrm{HCO}_{3}{ }^{-}$; et les sels nutritifs $\left(\mathrm{NO}_{3}{ }^{-}\right.$, $\mathrm{NO}_{2}{ }^{-}$et $\mathrm{NH}_{4}{ }^{+}$). Ces analyses ont été réalisées à l'aide d'un spectrophotomètre DR 2010 de type $\mathrm{HACH}$. Sur le terrain, les coordonnées des différents points d'eau ont été prises à l'aide d'un Global Positionning System (GPS) de type Garmin. Les coupes techniques de forages ont permis de déterminer la profondeur totale (PT) des forages et l'épaisseur des altérites (Ep-Alt) traversées lors de la foration. L'ensemble des données collectées sur les eaux souterraines de la région a fait l'objet d'une étude hydrochimique et statistique. Du point de vue hydrochimique, la méthode de classification chimique des eaux, basée sur une représentation graphique des données a été utilisée. Le diagramme triangulaire de Piper a été utilisé pour la réalisation de cette classification. L'approche de la statistique multivariée a été réalisée à partir de l'Analyse en Composantes Principales (ACP) sur des variables centrées réduites et de la Classification Ascendante Hiérarchique (CAH). Cette analyse statistique a pour but de comprendre l'hydrochimie des eaux souterraines. En effet, l'ACP est une méthode statistique multidimensionnelle descriptive, utilisable comme outil d'aide à l'interprétation d'une matrice de données. Cette analyse permet de synthétiser et de classer un nombre important de données afin d'en extraire les principaux facteurs qui sont à l'origine de l'évolution simultanée des variables et de leur relation réciproque (Biémi, 1992). Elle permet de mettre en évidence les ressemblances et la position graphique que présenteraient deux ou plusieurs variables chimiques au cours de leur évolution. La Classification Ascendante Hiérachique $(\mathrm{CAH})$ est un outil pour analyser des données de la chimie de l'eau et pour la formulation de modèles géochimiques (Yidana et al., 2008). C'est un système de classification qui utilise la distance euclidienne pour des mesures de similitude et la méthode de la tutelle pour lien qui produit la classification la plus distinctive où chaque membre dans un groupe est plus semblable à ses collègues qu'à n'importe quel membre à l'extérieur du groupe (Güler et al., 2002). L'analyse statistique multivariée a été réalisée sur 42 échantillons et 15 variables. Les paramètres utilisés pour cette analyse sont : la profondeur totale des ouvrages (PT), la minéralisation totale (MT), la conductivité électrique (CE), le $\mathrm{pH}$ et le $\Delta \mathrm{pH}$; les cations majeurs tels que $\mathrm{Ca}^{2+}, \mathrm{K}^{+}, \mathrm{Na}^{+}$et $\mathrm{Mg}^{2+}$; les anions majeurs dont le $\mathrm{Cl}^{-}, \mathrm{HCO}_{3}^{-}$, et le $\mathrm{SO}_{4}{ }^{2-}$ et les éléments nutritifs $\left(\mathrm{NO}_{3}^{-}\right.$et $\left.\mathrm{NO}_{2}^{-}\right)$. L'analyse statistique a été réalisée à l'aide du logiciel STATISTICA 6.0. L'utilisation de ces méthodes permet de connaître l'origine de la minéralisation des eaux des nappes de fractures et de déterminer les différents processus qui gouvernent la minéralisation des eaux souterraines de la région d'AbidjanAgboville .

\section{RESULTATS}

Les résultats des analyses physicochimiques réalisées sur les eaux souterraines de la région d'Abidjan-Agboville sont présentés dans le Tableau 1. La température des eaux souterraines varie entre $25,6{ }^{\circ} \mathrm{C}$ et $29,2{ }^{\circ} \mathrm{C}$, avec une moyenne de $27,7 \pm 0,1{ }^{\circ} \mathrm{C}$. Le $\mathrm{pH}$ des eaux varie entre 3,8 et 6,9 unités $\mathrm{pH}$, avec une moyenne de $5,8 \pm 0,1$; ce qui indique que les eaux souterraines de la région sont agressives. La conductivité électrique des eaux souterraines de la région est faible et varie entre 31,5 et $819 \mu \mathrm{S} . \mathrm{cm}^{-1}$, avec une 
valeur moyenne de 245,21 $\pm 22 \mu \mathrm{S} . \mathrm{cm}^{-1}$. Les eaux à forte conductivité sont celles des localités de Gori M'po (819 $\left.\mu \mathrm{S} . \mathrm{cm}^{-1}\right)$ et d'Ottopé $2\left(501 \mu \mathrm{S} . \mathrm{cm}^{-1}\right)$ à Agboville ainsi qu'à Arounakro $\left(572 \mu \mathrm{S} . \mathrm{cm}^{-1}\right)$ à Alépé. La dureté des eaux souterraines de la région varie entre $1{ }^{\circ} \mathrm{F}$ et $30^{\circ} \mathrm{F}$, soit une moyenne de $6,4 \pm$ $0,9^{\circ} \mathrm{F}$. Cela indique que les eaux de la région sont dans l'ensemble douces à l'exception de celles des localités de Gori M'po et d'Offa 2.

La représentation des résultats d'analyses chimiques dans le diagramme de Piper permet d'identifier quatre familles d'eau (Figure 3). Les eaux bicarbonatées calciques (C2) sont les plus importantes et représentent $61 \%$ des eaux étudiées. Elles se rencontrent principalement au niveau des eaux des forages des localités telles que Arraguié, Copa, Odoguié F2, Andé M'po, Boka M'po, Seguié, Attéhou F2, Ehouégué F2, Dibon M'po, Lapo, Omouho-M'po, Offa 2, M'bérie, M'battra, Agouahin et Gori M'po. Les eaux chlorurées sodi-potassiques $(22 \%)$ ne s'observent que dans les eaux des puits de Yakassémé, d'Agou, de Grand Morié et d'Ery-Makouguié. Ensuite viennent les eaux bicarbonatées sodipotassiques $(10 \%)$, rencontrées à Lobo-Opé, Diasson, Yapo-Gare et Bécédi Brignan et les eaux chlorurées calciques $(7 \%)$ observées à N'Cho M'po, Ekissi- Ho et d'Annépé. Dans la région, les eaux sont donc caractérisées par une prédominance des ions bicarbonates sur les ions chlorures et les sulfates qui sont pratiquement absents dans ces eaux. Le $\mathrm{Ca}^{2+}$ constitue le cation le plus important, puis viennent ensuite $\mathrm{Na}^{+}$et $\mathrm{K}^{+}$. Les eaux de la région présentent une hétérogénéité chimique dans leur ensemble.

Les résultats de l'ACP sont donnés par les Tableaux 2, 3 et 4. Le Tableau 2 montre que le $\mathrm{HCO}_{3}{ }^{-}$et le $\mathrm{Ca}^{2+}$, avec une moyenne respective de 74,2 $\pm 11,2 \mathrm{mg} . \mathrm{L}^{-1}$ et $15,6 \pm 3$ mg. $\mathrm{L}^{-1}$ constituent l'essentiel de la minéralisation totale des eaux souterraines. Le Tableau 3 présente les valeurs propres, les variances exprimées pour chaque facteur et leur cumul. Le facteur F1, avec une variance exprimée de 49,6\%, est le plus important de tous, ensuite viennent les facteurs F2 et F3, avec respectivement $19,39 \%$ et $10,19 \%$ de la variance exprimée. Les liens significatifs qui existent entre les différents paramètres sont donnés par la matrice de corrélation (Tableau 4). Celle-ci montre qu'il existe une importante corrélation entre $\mathrm{HCO}_{3}^{-}$et $\mathrm{Ca}^{2+}(\mathrm{r}=0,90)$. Aussi, le $\mathrm{Ca}^{2+}$ est-il bien corrélé avec MT $(\mathrm{r}=$ $0,96), \mathrm{CE}(\mathrm{r}=0,78), \mathrm{SO}_{4}{ }^{2-}(\mathrm{r}=0,94)$ et $\Delta \mathrm{pH}(\mathrm{r}$ $=0,71)$. Cette bonne corrélation s'observe également entre $\mathrm{pH}$ et $\Delta \mathrm{pH}(\mathrm{r}=0,93), \mathrm{K}^{+}(\mathrm{r}=$ $0,77), \mathrm{Na}^{+}(\mathrm{r}=0,77)$. L'analyse des résultats de l'ACP de l'espace des variables du plan factoriel F1-F2 (Figure 4) montre que ce plan factoriel F1-F2 exprime $69,01 \%$ de la variance exprimée. Le facteur $\mathrm{F} 1$, le plus important $(49,62 \%)$ est déterminé par MT, CE, $\Delta \mathrm{pH}$, $\mathrm{pH}, \mathrm{HCO}_{3}^{-}, \mathrm{Ca}^{2+}, \mathrm{Mg}^{2+}, \mathrm{SO}_{4}{ }^{2-}$ et $\mathrm{Cl}^{-}$. Les éléments qui définissent ce facteur proviennent d'une longue durée de mise en solution suite au contact «eau-roche». Ces éléments qui présentent des variations continues sur de longues périodes caractérisent la minéralisation de l'eau dans son encaissant. La forte corrélation qui existe entre ces différentes variables et leur proximité dans le cercle de communauté montrent que la mise en solution de ces différents ions est régie par le même phénomène qui est l'hydrolyse. En effet, l'hydrolyse étant un processus lent, il revient que le facteur F1 rende compte des conditions d'acquisition du chimisme de l'eau. Le facteur F1 exprime donc le phénomène de minéralisation-temps de séjour. Le regroupement de la majorité des variables soutenues par la minéralisation totale (MT), autour de cet axe montre l'influence de l'altération-hydrolyse dans la mise en solution des ions.

Le facteur 2 explique à lui seul 19,39\% de l'inertie du nuage des points représentatifs des ouvrages. Il est déterminé par $\mathrm{NO}_{3}{ }^{-}, \mathrm{NO}_{2}{ }^{-}$, $\mathrm{Na}^{+}, \mathrm{K}^{+}$et $\mathrm{pCO}_{2}$. La pression partielle de $\mathrm{CO}_{2}$ $\left(\mathrm{pCO}_{2}\right)$ s'oppose également au $\mathrm{pH}$ des eaux souterraines, cependant il apparaît une bonne corrélation entre ces deux variables (Figure 4). En effet, l'angle entre ces deux éléments est 
de $132^{\circ}$ (Figure 4 ). Le cosinus de cet angle, qui représente le coefficient de corrélation, est de $r=-0,68$. Cela montre que le facteur F2 qui est défini par ces variables est indépendant de l'hydrolyse. Ces variables s'opposent à la profondeur totale des ouvrages (PT); ce qui traduit l'intervention de la profondeur des ouvrages dans la mise en circulation de ces ions. Ainsi, la corrélation négative entre PT et $\mathrm{NO}_{3}^{-}(\mathrm{r}=-0,67), \mathrm{Na}^{+}(\mathrm{r}=-0,65)$ et $\mathrm{K}^{+}(\mathrm{r}=$ $0,66)$ montre que l'infiltration superficielle diminue avec la profondeur totale des ouvrages. Les composés azotés proviennent de la dégradation de la matière organique par les micro-organismes dans les couches superficielles du sol, avec production de $\mathrm{CO}_{2}$ pour être entraînés ensuite en profondeur par les eaux d'infiltration. Le facteur F2 exprime donc l'origine spatiale des ions. La position du PT a mi-chemin des facteurs F1 et F2 dans le cercle de communauté montre que la minéralisation de l'ensemble des eaux souterraines est influencée par la profondeur totale des ouvrages hydrauliques.

La représentation graphique dans l'espace des unités statistiques du plan factoriel F1-F2 (Figure 4) met en évidence trois principaux regroupements des points d'eau.

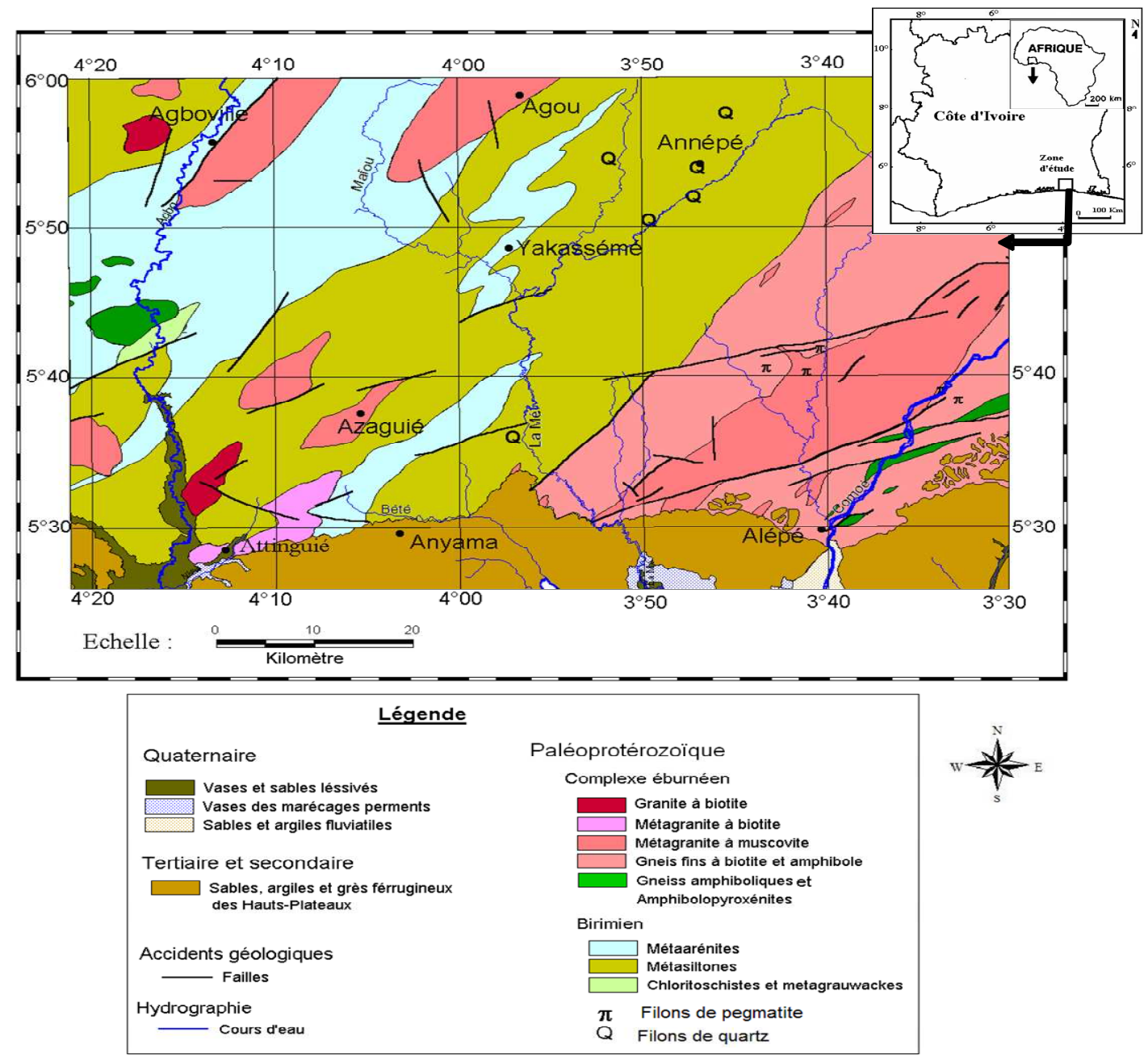

Figure 1 : Carte géologique de la région d'Abidjan-Agboville (modifiée de Delor et al., 1992). 


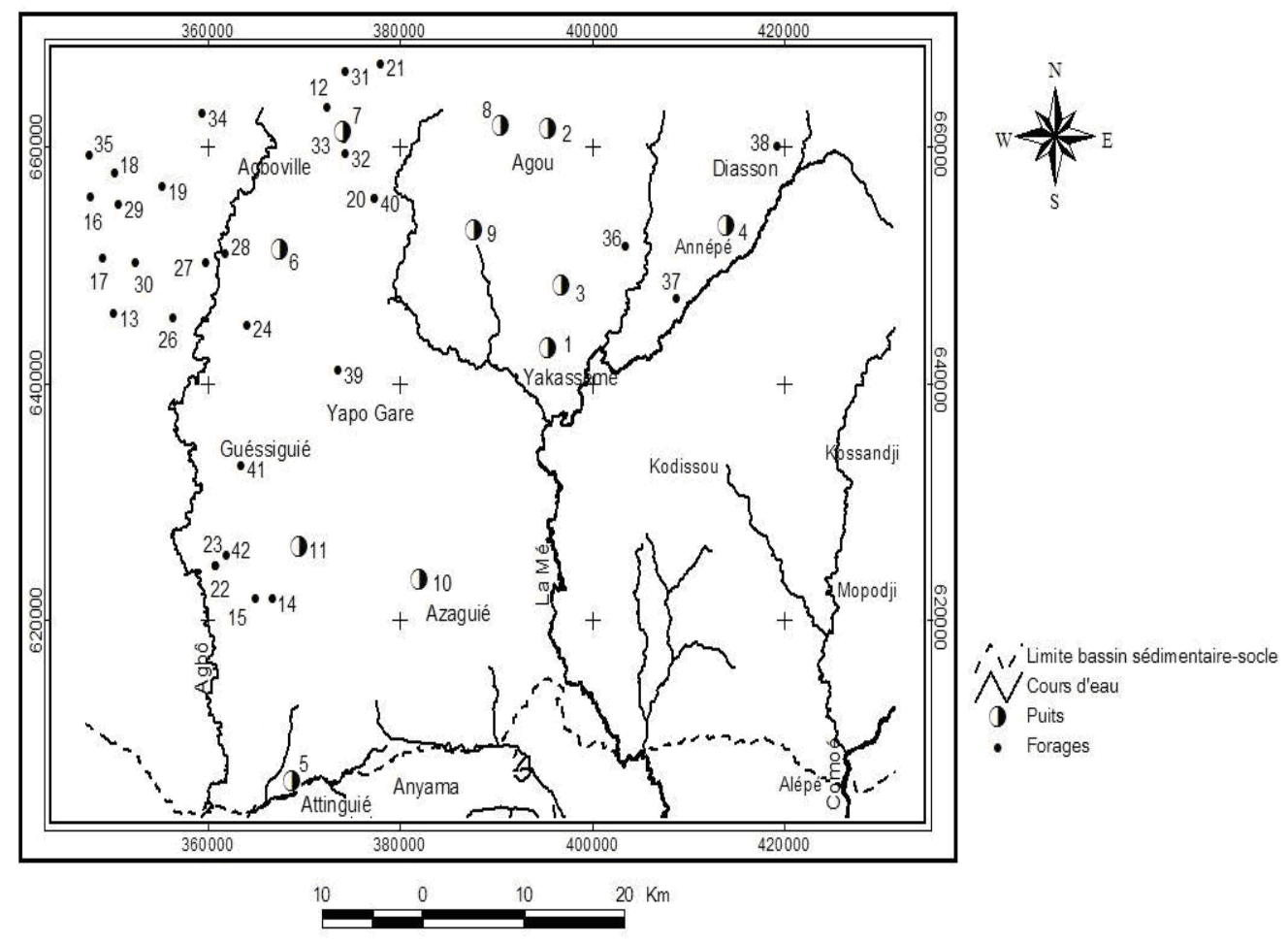

Figure 2 : Carte des points d'échantillonnage.

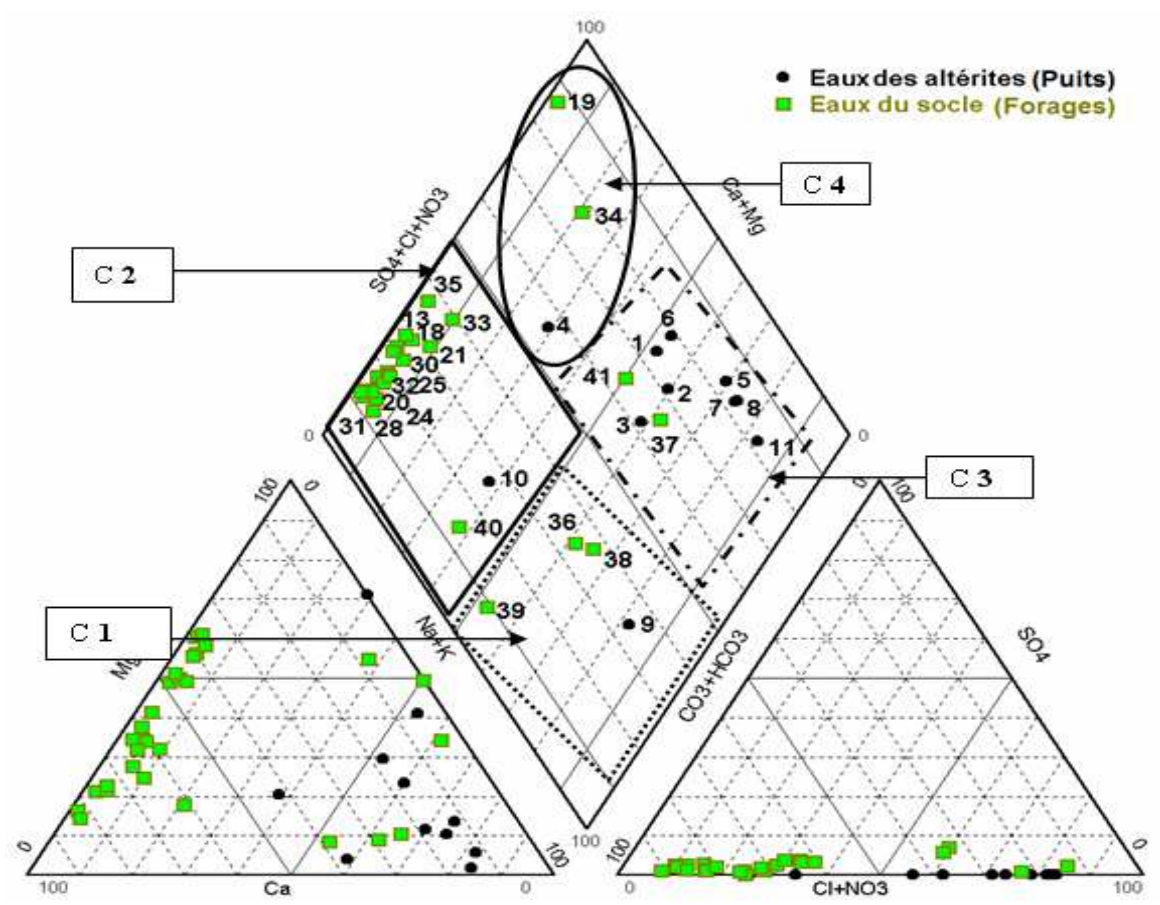

Figure 3 : Classification hydrochimique des eaux du socle dans le diagramme de Piper. 


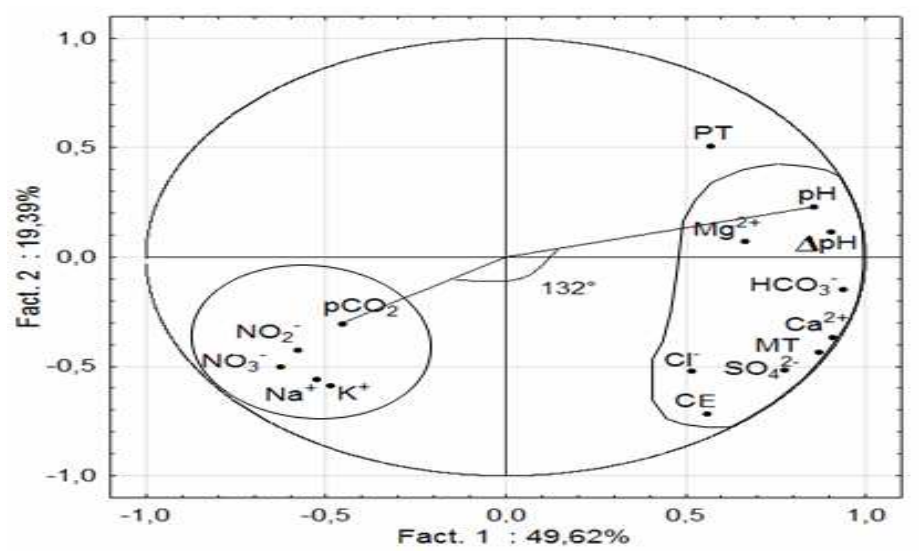

a) Espace des variables du plan factoriel F1 - F2

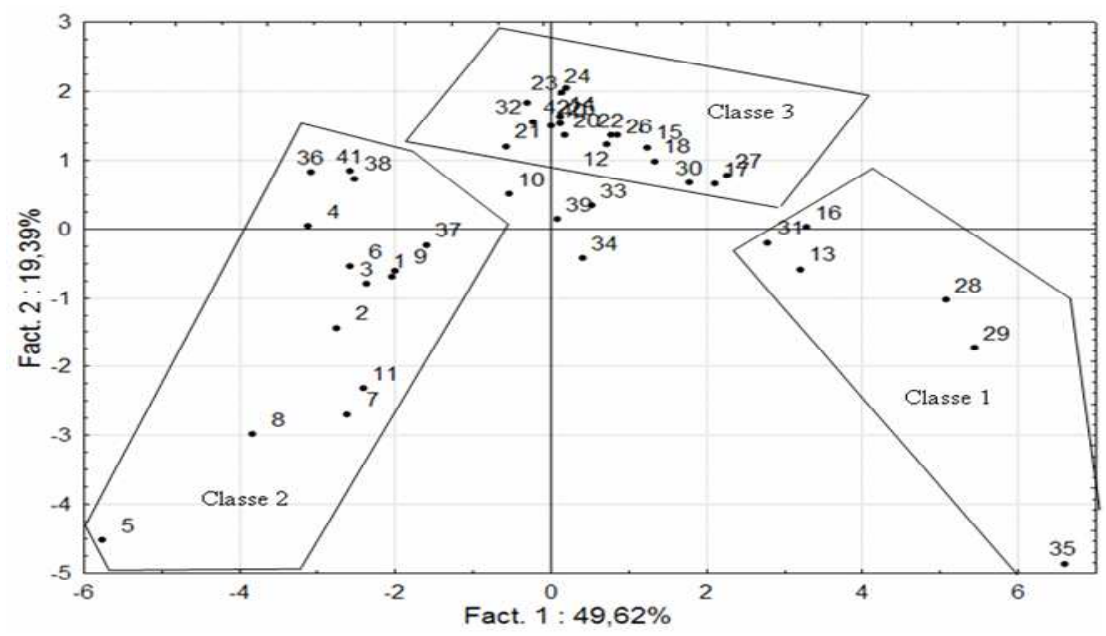

b) Espace des unités statistiques du plan factoriel F1 - F2

Figure 4 : Analyse en composantes principales normées des eaux du socle du plan factoriel F1 -F2.

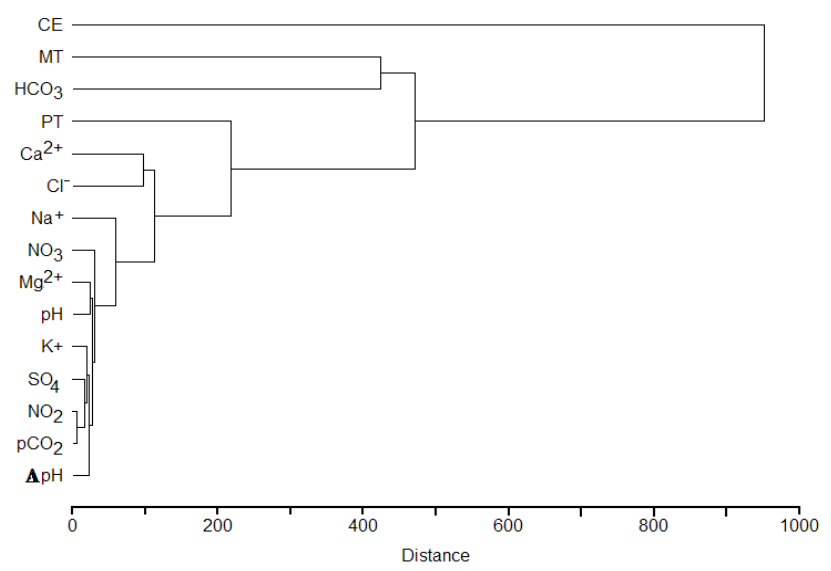

Figure 5 : Dendrogramme développé issu de la Classification Ascendante Hiérarchique (CAH). 
Tableau 1 : Données physico-chimiques en $\mathrm{mg} \cdot \mathrm{L}^{-1}$ des eaux souterraines et caractéristiques hydrauliques des forages de la région d'Abidjan-Agboville.

\begin{tabular}{|c|c|c|c|c|c|c|c|c|c|c|c|c|c|c|c|c|c|c|c|}
\hline & Localités & $\Delta \mathbf{p H}$ & $\begin{array}{c}\text { pCO2 } \\
\text { éq.[atm] }\end{array}$ & $\begin{array}{l}\text { PT } \\
(\mathbf{m})\end{array}$ & $\begin{array}{c}\text { Ep-Alt } \\
\text { (m) }\end{array}$ & $\mathbf{T}^{\circ}(\mathbf{C})$ & pH & 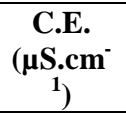 & $\mathrm{HCO}_{3}^{-}$ & $\mathrm{Cl}^{-}$ & $\mathrm{NO}_{3}^{-}$ & $\mathrm{NO}_{2}^{-}$ & $\mathbf{N H}_{4}{ }^{+}$ & $\mathrm{SO}_{2}^{4-}$ & $\mathrm{Ca}^{2+}$ & $\mathbf{M g}^{2+}$ & $\mathrm{Na}^{+}$ & $\mathbf{K}^{+}$ & MT \\
\hline 1 & Yakassémé & $-3,99$ & 0,04 & 7,3 & 6,8 & 28,8 & 5,5 & 204 & 11 & 12,44 & 9,9 & 0,8 & 0,06 & 1 & 5,23 & 5,25 & 15,97 & 3,35 & 65,20 \\
\hline 2 & Agou & $-4,56$ & 0,12 & 3 & 3 & 27,7 & 5 & 247 & 10,8 & 10,08 & 9,9 & 1,1 & 0,07 & 1 & 4,91 & 4,15 & 16,4 & 6,46 & 65,02 \\
\hline 3 & Abié & $-3,94$ & 0,10 & 10 & 10 & 28 & 5,2 & 230 & 14,2 & 7,2 & 11 & 0,6 & 0,08 & 1 & 9,71 & 0,62 & 16,04 & 2,81 & 63,76 \\
\hline 4 & Annépé & $-6,05$ & 0,16 & 3 & 3 & 29 & 5,1 & 31,5 & 16,8 & 3,04 & 16,8 & 1 & 0,14 & 1 & 0,01 & 6,48 & 4,7 & 0,54 & 50,76 \\
\hline 5 & $\begin{array}{l}\text { Attinguié } \\
\text { Ery- }\end{array}$ & $-5,97$ & 1,42 & 7 & 7 & 27 & 3,8 & 360 & 8 & 11,04 & 21,8 & 6 & 0,16 & 1 & 4,32 & 1,60 & 15,66 & 4,76 & 74,47 \\
\hline 6 & Makouguié 1 & $-5,35$ & 0,04 & 4,78 & 4,28 & 27 & 5,1 & 173,1 & 4,4 & 6,88 & 4,5 & 1,2 & 0,08 & 1 & 1,53 & 7,30 & 14,67 & 5,87 & 47,56 \\
\hline 7 & Grand Morié & $-4,73$ & 0,06 & 8 & 8 & 27,5 & 5 & 408 & 5,8 & 14,72 & 2,23 & 0,7 & 0,07 & 1 & 6,39 & 4,31 & 38,03 & 11,34 & 84,74 \\
\hline 8 & Boudépé & $-5,39$ & 0,46 & 2,5 & 2,5 & 27,9 & 4,3 & 257 & 8 & 18,2 & 8,7 & 0,7 & 0,09 & 1 & 5,12 & 2,07 & 20,05 & 14,86 & 78,85 \\
\hline 9 & Becedi Brignan & $-3,43$ & 0,05 & 1,5 & 1,5 & 25,6 & 5,9 & 176,1 & 35 & 9,44 & 1,9 & 1,9 & 0,12 & 1 & 2,83 & 0,79 & 19,89 & 4,23 & 80,44 \\
\hline 10 & Azaguie & $-1,92$ & 0,01 & 20 & 20 & 27,6 & 6,7 & 166,4 & 35,7 & 3,68 & 6 & 0,7 & 0,05 & 1 & 13,40 & 3,93 & 13,09 & 1,18 & 78,92 \\
\hline 11 & Aké Béfiat & $-3,86$ & 0,03 & 10 & 10 & 27,6 & 5,6 & 314 & 10,3 & 22,72 & 7,5 & 1,2 & 0,11 & 1 & 6,90 & 0,46 & 41,5 & 5,25 & 97,05 \\
\hline 12 & Arraguié & $-2,22$ & 0,07 & 54 & 29,8 & 28,3 & 6,1 & 216 & 79,3 & 17,73 & 0,5 & 0 & 0 & 1 & 12,83 & 4,37 & 0,95 & 0,35 & 124,37 \\
\hline 13 & Copa & $-1,51$ & 0,13 & 51,42 & 21,36 & 26,5 & 6,1 & 378 & 158,6 & 33,69 & 1,2 & 0 & 0 & 3 & 36,87 & 14,09 & 1,92 & 0,58 & 256,94 \\
\hline 14 & Odoguié F1 & $-2,71$ & 0,07 & 45 & 20 & 29,2 & 5,9 & 167,6 & 54,9 & 7,09 & 0,4 & 0 & 0 & 1 & 8,02 & 6,80 & 0,72 & 0,42 & 89,48 \\
\hline 15 & Odoguié F2 & $-1,68$ & 0,04 & 40,5 & 18 & 26,1 & 6,5 & 204 & 103,7 & 7,09 & 0,5 & 0 & 0 & 2 & 15,23 & 6,80 & 0,72 & 0,42 & 143,64 \\
\hline 16 & Andé M'po & $-0,86$ & 0,05 & 63 & 16,5 & 29 & 6,6 & 380 & 183 & 14,18 & 0,9 & 0 & 0 & 3 & 40,88 & 6,80 & 1,27 & 0,42 & 257,56 \\
\hline 17 & Edi M'po & $-1,76$ & 0,08 & 40,5 & 16 & 27,6 & 6,2 & 225 & 115,9 & 12,41 & 0,6 & 0 & 0 & 3 & 20,04 & 16,52 & 1,82 & 0,79 & 178,30 \\
\hline 18 & Boka M'po & $-1,98$ & 0,08 & 45 & 18 & 27,1 & 6,1 & 228 & 97,6 & 17,73 & 0,8 & 0 & 0 & 1 & 17,64 & 10,69 & 0,92 & 0,36 & 153,65 \\
\hline 19 & Ekissi ho & $-2,81$ & 0,01 & & 18 & 28,3 & 6,5 & 268 & 7,8 & 28,37 & 0,5 & 0 & 0 & 1 & 12,02 & 11,66 & 0,68 & 0,25 & 69,78 \\
\hline 20 & Lapo & $-2,76$ & 0,23 & 49,5 & 36 & 28,9 & 5,6 & 153,5 & 79,3 & 8,87 & 0,5 & 0 & 0 & 1 & 11,22 & 7,29 & 0,9 & 0,52 & 116,80 \\
\hline 21 & Segué & $-3,42$ & 0,21 & 49,5 & 23 & 27,3 & 5,3 & 217 & 36,6 & 8,87 & 0,4 & 0 & 0 & 1 & 10,42 & 3,40 & 1,52 & 0,67 & 70,14 \\
\hline 22 & Attéhou F2 & $-2,06$ & 0,08 & 45 & 19 & 26,5 & 6,1 & 157,7 & 91,5 & 10,64 & 0,3 & 0 & 0 & 1 & 17,64 & 5,83 & 0,78 & 0,25 & 134,92 \\
\hline 23 & Attéhou F1 & $-2,59$ & 0,05 & 45 & 24 & 27,5 & 6,1 & 111,2 & 54,9 & 3,55 & 0,3 & 0 & 0 & 1 & 7,21 & 7,29 & 0,56 & 0,1 & 81,92 \\
\hline
\end{tabular}


E. K. AHOUSSI et al. / Int. J. Biol. Chem. Sci. 4(3):782-797, 2010

\begin{tabular}{|c|c|c|c|c|c|c|c|c|c|c|c|c|c|c|c|c|c|c|c|}
\hline 24 & Ehouégué F2 & $-2,49$ & 0,06 & 72 & 26 & 27,6 & 6,1 & 136,5 & 54,9 & 7,09 & 0,2 & 0 & 0 & 1 & 11,22 & 1,94 & 0,65 & 0,28 & 84,26 \\
\hline 25 & Dibon M'po & $-2,51$ & 0,11 & 50 & 25 & 27,4 & 5,8 & 149,8 & 67,1 & 12,41 & 0,5 & 0 & 0 & 0 & 14,43 & 4,86 & 1,25 & 0,56 & 108,29 \\
\hline 26 & Gbéssé & $-2,3$ & 0,11 & 46,92 & 18,29 & 28,6 & 6 & 173,9 & 91,5 & 10,64 & 0,4 & 0 & 0 & 1 & 11,22 & 10,69 & 1,26 & 0,73 & 134,89 \\
\hline 27 & Offoriguié & $-1,55$ & 0,06 & 54,86 & 23,4 & 27,7 & 6,4 & 266 & 128,1 & 10,64 & 0,3 & 0 & 0 & 3 & 19,24 & 12,64 & 2,36 & 0,7 & 184,17 \\
\hline 28 & Omouho-M'po & $-0,54$ & 0,06 & 67,5 & 21,4 & 27,1 & 6,7 & 370 & 274,5 & 17,73 & 0,9 & 0 & 0 & 6 & 50,50 & 12,64 & 5,12 & 1,2 & 376,13 \\
\hline 29 & Offa 2 & $-0,32$ & 0,04 & 49,5 & 18 & 27,2 & 6,8 & 501 & 256,2 & 21,28 & 0,5 & 0 & 0 & 6 & 65,73 & 12,15 & 3,67 & 0,9 & 373,37 \\
\hline 30 & Badoukro & $-1,65$ & 0,05 & 37,08 & 18,37 & 27,3 & 6,4 & 287 & 122 & 21,28 & 0,3 & 0 & 0 & 1 & 16,03 & 10,69 & 0,82 & 0,45 & 179,76 \\
\hline 31 & M'Bérie & $-1,06$ & 0,06 & 45,54 & 13,66 & 26,4 & 6,5 & 395 & 183 & 12,41 & 1,2 & 0 & 0 & 3 & 36,87 & 4,37 & 0,78 & 0,25 & 249,10 \\
\hline 32 & M'Battra & $-3,13$ & 0,18 & 67,5 & 26 & 27,7 & 5,5 & 135,4 & 54,9 & 7,09 & 0,4 & 0 & 0 & 1 & 8,02 & 2,43 & 0,59 & 0,3 & 82,10 \\
\hline 33 & Agouahin & $-2,77$ & 0,25 & 51,67 & 8,65 & 27,6 & 5,5 & 227 & 73,2 & 24,82 & 0,7 & 0 & 0 & 3 & 15,23 & 3,40 & 2,19 & 0,73 & 130,68 \\
\hline 34 & N'Cho M'po & $-3,2$ & 0,12 & 41,67 & 10,96 & 27,2 & 5,5 & 262 & 36,6 & 70,92 & 0,8 & 0 & 0 & 1 & 11,22 & 1,94 & 3,95 & 0,8 & 134,26 \\
\hline 35 & Gori M'po & $-0,07$ & 0,03 & 49,93 & 3,74 & 26,6 & 6,9 & 819 & 268,4 & 81,56 & 1,5 & 0 & 0 & 12 & 102,61 & 10,69 & 3,56 & 0,95 & 488,66 \\
\hline 36 & Lobo Opé & $-6,02$ & 1,06 & 74,66 & 42,62 & 27 & 4,3 & 41,9 & 20 & 4 & 2,06 & 1,9 & 0,13 & 1 & 0,43 & 2,07 & 6,95 & 0,19 & 40,26 \\
\hline 37 & Lobo-Akouzin & $-4,72$ & 0,04 & 35 & 20 & 28,4 & 5,3 & 503 & 6,3 & 5,12 & 2,5 & 1,1 & 0,07 & 1 & 2,75 & 0,50 & 5,15 & 2,82 & 29,24 \\
\hline 38 & Diasson & $-4,64$ & 0,11 & 30 & 30 & 28,8 & 5,2 & 94,3 & 15,1 & 2,88 & 3,1 & 2,7 & 0,11 & 1 & 1,66 & 0,43 & 4,85 & 0,66 & 32,73 \\
\hline 39 & Yapo Gare & $-1,87$ & 0,04 & 53,06 & 27,37 & 28 & 6,4 & 263 & 108,3 & 5,44 & 0,4 & 1,9 & 1,35 & 1 & 9,73 & 1,25 & 13,41 & 3,31 & 158,45 \\
\hline 40 & Lapo & $-3,21$ & 0,05 & 75,75 & 29,5 & 28 & 6,1 & 170,4 & 64,2 & 4,8 & 1,4 & 0,8 & 0,18 & 1 & 1,6 & 6,71 & 7,99 & 1,35 & 116,13 \\
\hline 41 & Guéssiguié & $-6,94$ & 0,16 & 54,5 & 23,4 & 27,6 & 4,7 & 97,5 & 8 & 3,2 & 8,1 & 0,4 & 0,09 & 1 & 0,05 & 6,26 & 11,6 & 0,95 & 39,90 \\
\hline 42 & Attéhou & $-2,78$ & 0,06 & 45 & 21,5 & 29,2 & 6,1 & 133,1 & 59,5 & 1,6 & 1,3 & 1 & 0,09 & 1 & 4,43 & 8,17 & 3,57 & 0,42 & 81,55 \\
\hline
\end{tabular}

Tableau 2 : Variables et valeurs extrêmes.

\begin{tabular}{lccccc}
\hline & Moyenne & Minimum & Maximum & Ecart-type & Erreur Standard sur la Moyenne (ESM) \\
\hline $\mathbf{\Delta} \mathbf{p H}$ & $-3,0$ & $-6,9$ & $-0,1$ & 1,7 & 0,3 \\
$\mathbf{p C O} \mathbf{2}_{\mathbf{2}}$ & 0,1 & 0,0 & 1,4 & 0,3 & 0,0 \\
$\mathbf{P t}$ & 39,2 & 1,5 & 75,8 & 22,2 & 3,5 \\
$\mathbf{T}\left({ }^{\circ} \mathbf{C}\right)$ & 27,7 & 25,6 & 29,2 & 0,9 & 0,1 \\
$\mathbf{p H}$ & 5,8 & 3,8 & 6,9 & 0,7 & 0,1 \\
$\mathbf{C . E .}$ & 245,2 & 31,5 & 819,0 & 142,6 & 22,0 \\
$\mathbf{H C O}_{3}^{-}$ & 74,2 & 4,4 & 274,5 & 72,7 & 11,2
\end{tabular}


E. K. AHOUSSI et al. / Int. J. Biol. Chem. Sci. 4(3):782-797, 2010

\begin{tabular}{lccccc}
$\mathbf{C l}^{-}$ & 14,6 & 1,6 & 81,6 & 15,8 & 2,4 \\
$\mathbf{N O}_{3}{ }^{-}$ & 3,2 & 0,2 & 21,8 & 4,8 & 0,7 \\
$\mathbf{N O}_{2}^{-}$ & 0,6 & 0,0 & 6,0 & 1,1 & 0,2 \\
$\mathbf{N H}_{4}{ }^{+}$ & 0,1 & 0,0 & 1,4 & 0,2 & 0,0 \\
$\mathbf{S O}_{4}{ }^{-}$ & 1,8 & 0,0 & 12,0 & 2,0 & 0,3 \\
$\mathbf{C a}^{2+}$ & 15,6 & 0,0 & 102,6 & 19,5 & 3,0 \\
$\mathbf{M g}^{2+}$ & 6,0 & 0,4 & 16,5 & 4,2 & 0,7 \\
$\mathbf{N a}^{+}$ & 7,3 & 0,6 & 41,5 & 9,5 & 1,5 \\
$\mathbf{K}^{+}$ & 2,0 & 0,1 & 14,9 & 3,0 & 0,5 \\
$\mathbf{M T}$ & 130,7 & 29,2 & 488,7 & 98,6 & 15,2 \\
Dureté & 6,4 & 1,0 & 30,0 & 5,9 & 0,9 \\
\hline
\end{tabular}

Tableau 3 : Valeurs propres et pourcentages des variances exprimées par les axes principaux.

\begin{tabular}{lccc}
\hline & F1 & F2 & F3 \\
\hline Valeur propre & 7,44 & 2,91 & 1,53 \\
\% de Variance exprimée & 49,62 & 19,39 & 10,19 \\
Cumul de valeur propre & 7,44 & 10,35 & 11,88 \\
\% de Variance exprimée cumulée & 49,62 & 69,01 & 79,20 \\
\hline
\end{tabular}




\section{E. K. AHOUSSI et al. / Int. J. Biol. Chem. Sci. 4(3):782-797, 2010}

Tableau 4 : Matrice de corrélation.

\begin{tabular}{|c|c|c|c|c|c|c|c|c|c|c|c|c|c|c|c|}
\hline & $\Delta \mathrm{pH}$ & $\mathrm{pCO}_{2}$ & PT & pH & $\mathrm{CE}$ & $\mathrm{HCO}_{3}^{-}$ & $\mathrm{Cl}^{-}$ & $\mathrm{NO}_{3}{ }^{-}$ & $\mathrm{NO}_{2}^{-}$ & $\mathrm{SO}_{4}{ }^{2-}$ & $\mathrm{Ca}^{2+}$ & $\mathrm{Mg}^{2+}$ & $\mathbf{N a}^{+}$ & $\mathbf{K}^{+}$ & MT \\
\hline$\Delta \mathrm{pH}$ & 1,00 & & & & & & & & & & & & & & \\
\hline $\mathrm{pCO}_{2}$ & $\begin{array}{c}- \\
0,48\end{array}$ & 1,00 & & & & & & & & & & & & & \\
\hline PT & 0,48 & $-0,07$ & 1,00 & & & & & & & & & & & & \\
\hline pH & 0,93 & $-0,68$ & 0,43 & 1,00 & & & & & & & & & & & \\
\hline CE & 0,43 & $-0,09$ & $\begin{array}{c}- \\
0,01\end{array}$ & 0,32 & 1,00 & & & & & & & & & & \\
\hline $\mathrm{HCO}_{3}{ }^{-}$ & 0,84 & $-0,26$ & 0,50 & 0,76 & 0,59 & 1,00 & & & & & & & & & \\
\hline $\mathrm{Cl}^{-}$ & 0,35 & $-0,08$ & 0,06 & 0,22 & 0,64 & 0,42 & 1,00 & & & & & & & & \\
\hline $\mathrm{NO}_{3}{ }^{-}$ & $\begin{array}{c}- \\
0,65\end{array}$ & 0,52 & $\begin{array}{c}- \\
0,67\end{array}$ & $\begin{array}{c}- \\
0,64\end{array}$ & $-0,07$ & $-0,46$ & $\begin{array}{c}- \\
0,15\end{array}$ & 1,00 & & & & & & & \\
\hline $\mathrm{NO}_{2}^{-}$ & $\begin{array}{c}- \\
0,55\end{array}$ & 0,70 & $\begin{array}{c}- \\
0,43\end{array}$ & $\begin{array}{c}- \\
0,59\end{array}$ & $-0,05$ & $-0,41$ & $\begin{array}{c}- \\
0,22\end{array}$ & 0,66 & 1,00 & & & & & & \\
\hline $\mathrm{SO}_{4}{ }^{2-}$ & 0,55 & $-0,13$ & 0,24 & 0,49 & 0,76 & 0,80 & 0,64 & $\begin{array}{c}- \\
0,19\end{array}$ & $\begin{array}{c}- \\
0,23\end{array}$ & 1,00 & & & & & \\
\hline $\mathrm{Ca}^{2+}$ & 0,71 & $-0,20$ & 0,31 & 0,61 & 0,78 & 0,90 & 0,64 & $\begin{array}{c}- \\
0,28\end{array}$ & $\begin{array}{c}- \\
0,34\end{array}$ & 0,94 & 1,00 & & & & \\
\hline $\mathbf{M g}^{2+}$ & 0,54 & $-0,26$ & 0,30 & 0,53 & 0,23 & 0,65 & 0,19 & $\begin{array}{c}- \\
0,32\end{array}$ & $\begin{array}{c}- \\
0,45\end{array}$ & 0,48 & 0,51 & 1,00 & & & \\
\hline $\mathrm{Na}^{+}$ & $\begin{array}{c}- \\
0,47\end{array}$ & 0,09 & $\begin{array}{c}- \\
0,65\end{array}$ & $\begin{array}{c}- \\
0,41\end{array}$ & 0,11 & $-0,44$ & $\begin{array}{c}- \\
0,04\end{array}$ & 0,46 & 0,41 & $-0,18$ & $\begin{array}{c}- \\
0,27\end{array}$ & $-0,41$ & 1,00 & & \\
\hline $\mathbf{K}^{+}$ & $\begin{array}{c}- \\
0,45\end{array}$ & 0,17 & $\begin{array}{c}- \\
0,66\end{array}$ & $\begin{array}{c}- \\
0,51\end{array}$ & 0,17 & $-0,38$ & $\begin{array}{c}- \\
0,01\end{array}$ & 0,40 & 0,31 & $-0,15$ & $\begin{array}{c}- \\
0,23\end{array}$ & $-0,32$ & 0,77 & 1,00 & \\
\hline MT & 0,78 & $-0,22$ & 0,37 & 0,68 & 0,73 & 0,96 & 0,61 & $\begin{array}{c}- \\
0,35\end{array}$ & $\begin{array}{c}- \\
0,35\end{array}$ & 0,89 & 0,96 & 0,60 & $\begin{array}{c}- \\
0,28\end{array}$ & $\begin{array}{c}- \\
0,24\end{array}$ & 1,00 \\
\hline
\end{tabular}


La classe 1 contient les eaux fortement minéralisées (14\%). Ces points d'eau correspondent aux forages profonds qui captent le socle fracturé. Ces eaux sont riches en bicarbonates, avec des teneurs de 274, 50 mg.L. $\mathrm{L}^{-1}$ à Omouho M'po, 268,4 mg.L $\mathrm{L}^{-1}$ à Gori M'po, 256,20 mg.L à Offa $2 ; 183 \mathrm{mg} . \mathrm{L}^{-1}$ à Andé M'po et M'bérié et 158,60 mg. $\mathrm{L}^{-1}$ à Copa dans la région d'Agboville. La classe 2 regroupe les eaux moyennement minéralisées et ayant une forte teneur en nitrates (33\%). Elle renferme l'ensemble des eaux de puits. Il s'agit respectivement des puits des localités de Yakassémé, d'Agou, d'Abié, d'Annépé, d'Attinguié, de Grand Morié, d'EryMakouguié, de Boudépé, de Bécédi Brignan et d'Aké Béfiat. La classe 3 contient les eaux faiblement minéralisées de la région (48\%), soit l'essentiel des points d'eau étudiés. Ces forages sont caractérisés par une profondeur moyenne, avec une faible teneur en $\mathrm{HCO}_{3}{ }^{-}$.

Le dendrogramme (Figure 5) issu de la Classification Ascendante Hiérarchique (CAH) met en évidence deux principaux regroupements des variables. Le premier regroupement est constitué de $\mathrm{CE}, \mathrm{MT}, \mathrm{HCO}_{3}^{-}$ $, \mathrm{PT}, \mathrm{Ca}^{2+}$ et $\mathrm{Cl}^{-}$. Ce groupe rend compte de la minéralisation-temps de séjour ou du phénomène de l'hydrolyse des minéraux silicatés. Le second groupe constitué de $\mathrm{Na}^{+}$, $\mathrm{NO}_{3}{ }^{-}, \mathrm{Mg}^{2+}, \mathrm{pH}, \mathrm{K}^{+}, \mathrm{SO}_{4}{ }^{2-}, \mathrm{NO}_{2}{ }^{-}, \mathrm{pCO}_{2}$ et $\Delta \mathrm{pH}$ indique la contribution des activités anthropiques dans la minéralisation des eaux souterraines de la région. Ce groupe met également en évidence le phénomène de minéralisation gouverné par l'infiltration des eaux de pluie dans les aquifères.

\section{DISCUSSION}

L'Analyse en Composantes Principales et la Classification Ascendante Hiérarchique donnent des résultats identiques sur l'origine de la minéralisation des eaux souterraines de la région. Celles-ci montrent que la minéralisation des eaux souterraines est contrôlée par la nature des formations géologiques présentes dans la région d'Abidjan-Agboville. En effet, l'origine des ions dans les eaux souterraines est contrôlée par le contact eau-roche comme l'indique les résultats des différentes études statistiques. La géologie de la région est dominée par les formations du Paléoprotérozoïque constituées principalement de granites, de gneiss et de schistes. L'hydrolyse de telles roches riches en feldspaths alcalins et en plagioclases acides, explique que les teneurs en $\mathrm{Ca}^{2+}$ et en $\mathrm{Na}^{+}$soient dominantes pour les cations dans les eaux souterraines. Ces ions sont issus de l'altération des roches et de l'hydrolyse des minéraux silicatés (Savané et al., 2001). La proportion assez importante d'anorthite dans les plagioclases, variété la plus facilement altérable (Faillat et Drogue, 1993) justifie que la concentration en $\mathrm{Ca}^{2+}\left(15,56 \pm 3,0 \mathrm{mg} . \mathrm{L}^{-1}\right)$ des eaux soit supérieure à celle en $\mathrm{Na}^{+}(7,34 \pm$ $\left.1,5 \mathrm{mg} . \mathrm{L}^{-1}\right)$. En effet, le calcium est inclus dans la structure des plagioclases. Selon Soro (2002), le calcium libéré, au cours de l'hydrolyse acide des plagioclases, évolue conjointement avec les bicarbonates dans les eaux selon la réaction 1 :

$$
\begin{aligned}
& \quad 5\left(\mathrm{Na}_{0.8}, \mathrm{Ca}_{0.2}\right)\left(\mathrm{Al}_{1.2} \mathrm{Si}_{2.8}, \mathrm{O}_{8}\right)+6 \mathrm{H}^{+}+ \\
& 19 \mathrm{H}_{2} \mathrm{O} \rightarrow 3 \mathrm{Al}_{2} \mathrm{O}_{5}(\mathrm{OH})_{4}+4 \mathrm{Na}^{+}+\mathrm{Ca}^{2+}+ \\
& 8 \mathrm{H}_{4} \mathrm{SiO}_{4}(1)
\end{aligned}
$$

Cette réaction est plus marquée dans les eaux souterraines de sorte que la corrélation entre le $\mathrm{Ca}^{2+}$ et le $\mathrm{HCO}_{3}{ }^{-}$est forte $(\mathrm{r}=0,90)$. $\mathrm{La}$ minéralisation calcique des eaux du socle est sous l'influence de l'altération et de l'hydrolyse des minéraux alumino-sillicates, riches en $\mathrm{Ca}^{2+}$ comme l'anorthite selon la réaction 2 :

$$
\begin{aligned}
& \mathrm{CaAl}_{2} \mathrm{Si}_{2} \mathrm{O}_{8}+2 \mathrm{H}_{2} \mathrm{CO}_{3}+\mathrm{H}_{2} \mathrm{O} \\
& +2 \mathrm{HCO}_{3}^{-}+\mathrm{Al}_{2} \mathrm{Si}_{2} \mathrm{O}_{5}(\mathrm{OH})_{4}
\end{aligned} \rightarrow \mathrm{Ca}^{2+}
$$

Le rapport $\mathrm{Mg}^{2+} / \mathrm{Ca}^{2+}$ reste, dans l'ensemble des points d'eau, inférieur à 1 ; ce qui indique que les ions $\mathrm{Mg}^{2+}$ sont issus de la décomposition des minéraux ferro-magnésiens tels que la biotite et l'amphibole présentes dans les roches de la région. Le rapport $\mathrm{Na}^{+} / \mathrm{K}^{+}$est dans la majorité des points d'eau supérieur à 1 ; ce qui signifie que ces deux ions évoluent différemment dans les eaux. La prédominance des ions $\mathrm{Na}^{+}$sur $\mathrm{K}^{+}$peut s'expliquer d'une part par la grande stabilité de la muscovite et des feldspaths potassiques 
et d'autre part l'adsorption des ions potassium par les plantes (Soro, 2002). La présence dans l'eau de $\mathrm{CO}_{2}$ provenant des sols facilite l'hydrolyse des silicates qui permet la formation de $\mathrm{HCO}^{-}$(Faillat et Drogue, 1993). C'est la raison pour laquelle l'eau de la nappe de fissure présente simultanément les plus fortes teneurs en $\mathrm{HCO}_{3}{ }^{-}\left(274,5 \mathrm{mg} . \mathrm{L}^{-1}\right)$ et les plus forts $\mathrm{pH}$ (proche de 7). En zone forestière, la mise en solution de cet ion dans les eaux est gouvernée par la pression partielle de $\mathrm{CO}_{2}$ biogénique (Soro, 2002). La région d'étude étant une zone de forêt dense sempervirente et ombrophile, c'est au cours de l'infiltration en profondeur des eaux de pluie dans ces terrains que l'eau chargée de $\mathrm{CO}_{2}$ va dissoudre les formations et s'enrichir en bicarbonates. C'est d'ailleurs, cette hydrolyse acide des roches encaissantes qui contrôle la diversité des faciès bicarbonatés des eaux souterraines. Les ions $\mathrm{Cl}^{-}, \mathrm{SO}_{4}{ }^{2-}$ et $\mathrm{NO}_{3}{ }^{-}$et une partie des cations, proviennent de la pluie et du lessivage des sols par celle-ci comme cela l'indique les résultats de l'analyse multivariée. En effet, les apports des eaux de pluies en $\mathrm{Cl}^{-}$ dans le Grand Abidjan, situé au Sud de la Côte d'Ivoire sont en moyenne $20 \mathrm{mg} . \mathrm{L}^{-1}$ (Oga, 1998). Ce qui est proche de la moyenne des eaux souterraines $\left(14,63 \pm 2,4 \mathrm{mg} . \mathrm{L}^{-1}\right)$ de la région d'Abidjan-Agboville. Cela montre que les eaux de pluies constituent la principale source de production d'ions $\mathrm{Cl}^{-}$dans les eaux souterraines. Les fortes teneurs en $\mathrm{NO}_{3}{ }^{-}$sont observées dans les eaux de puits. Selon Baali et al. (2007), Ahoussi et al. (2007, 2008 et 2009) et Ben Hamouda et al. (2009), ces ions $\mathrm{NO}_{3}{ }^{-}$sont issus d'une pollution d'origine humaine liée aux activités anthropiques. L'influence des activités anthropogéniques dans la minéralisation des eaux souterraines a été également mise en évidence dans les eaux souterraines par John et al. (2007) en Inde et Yidana (2010) au Ghana. Les faibles teneurs en sulfates rencontrées dans les eaux souterraines de la zone constituent une particularité des eaux souterraines de la Côte
d'Ivoire (Oga, 1998; Soro, 2002 ; Ahoussi, 2008). Selon ces auteurs, les eaux de pluie représentent la source de production des ions $\mathrm{SO}_{4}{ }^{2-}$ dans les eaux souterraines. En effet, pour Travi et Mudry (1997), les teneurs en chlorures et sulfates sont habituellement faibles dans les réservoirs du socle cristallin de l'Afrique de l'Ouest.

\section{Conclusion}

L'étude de l'origine de la minéralisation des eaux des aquifères discontinus de la région d'Agboville à partir de la combinaison des méthodes hydrochimiques graphiques et de l'analyse statistique multivariée a conduit à la mise en évidence des différentes caractéristiques des eaux souterraines de la région. Les eaux souterraines ont une température qui varie entre $25,6^{\circ} \mathrm{C}$ et $29,2{ }^{\circ} \mathrm{C}$, avec une moyenne de $27,7 \pm 0,1^{\circ} \mathrm{C}$. Elles sont agressives, avec un $\mathrm{pH}$ qui varie entre 3,8 et 6,9 unités $\mathrm{pH}$, pour une moyenne de $5,8 \pm 0,1$. Les eaux souterraines sont douces, avec une dureté qui varie entre $1^{\circ} \mathrm{F}$ et $30^{\circ} \mathrm{F}$. Dans la région, les eaux souterraines se regroupent en quatre principaux hydrofaciès. Les eaux bicarbonatées calciques sont les plus importantes et représentent $61 \%$ des eaux étudiées. Ensuite viennent les eaux chlorurées sodi-potassiques $(22 \%), \quad$ les eaux bicarbonatées sodi-potassiques $(10 \%)$ et les eaux chlorurées calciques (7\%). L'Analyse en Composantes Principales et la Classification Ascendante Hiérarchique indiquent que la minéralisation des eaux souterraines est contrôlée par la nature des formations géologiques présentes dans la région. Ainsi les principaux ions sont issus de l'altération des roches, de l'hydrolyse des minéraux silicatés comme l'anorthite dans les plagioclases, de la décomposition des minéraux ferro-magnésiens tels que la biotite et l'amphibole présentes dans les roches. Par ailleurs, c'est l'hydrolyse acide des roches encaissantes qui contrôle la diversité des faciès bicarbonatés des eaux 
souterraines. Les ions $\mathrm{Cl}^{-}$et $\mathrm{SO}_{4}{ }^{2-}$ proviennent de la pluie et du lessivage des sols. Les ions $\mathrm{NO}_{3}^{-}$sont issus d'une pollution d'origine humaine liée aux activités anthropiques.

\section{REFERENCES BIBLIOGRAPHIQUES}

Ahoussi KE, Soro N, Soro G, Kouadio FJL, Soro TD, Biémi J. 2007. Évaluation de la qualité physico-chimique des eaux de la nappe d'altérite captée par les puits servant à l'approvisionnement en eau des populations de la ville d'Agboville (Côte d'Ivoire). Journal Africain de Communication Scientifiques et Technologique, 2: 109-121.

Ahoussi KE. 2008. Évaluation quantitative et qualitative des ressources en eau dans le Sud de la Côte d'Ivoire. Application de l'hydrochimie et des isotopes de l'environnement à l'étude des aquifères continus et discontinus de la région Abidjan-Agboville. Thèse Université de Cocody, Abidjan, Côte d'Ivoire, p. 270.

Ahoussi KE, Soro N, Soro G, Lasm T, Oga MS, Zadé S. 2008. Groundwater Pollution in Africans Biggest Towns: Case of the Town of Abidjan (Côte d'Ivoire). European Journal of Scientific Research, 20(2): 302-316.

Ahoussi KE, Soro N, Soro G, Oga MS, Zadé S. 2009. Caractérisation physicochimique et bactériologique des eaux de puits de la ville d'Abidjan (Côte d'Ivoire). Africa Geoscience Review, 16(3): 203-2015.

Baali F, Rouabhia A, Kherici N, Djabri L, Bouchaou L, Hani A. 2007. Qualité des eaux souterraines et risque de pollution en milieu semi-aride. Cas de la cuvette de Chéria (NE Algérien). Estudios Geológicos, 63(2): 127-133

Ben Hamouda MF, Leduc C, Tarhouni J, Zouari K. 2009. Origine de la minéralisation dans l'aquifère plioquaternaire de la côte orientale du cap Bon (Tunisie). Sécheresse, 20(1): 78-86.
Biémi J. 1992. Contribution à l'étude géologique, hydrogéologique et par télédétection des bassins versants Subsahéliens du socle précambrien d'Afrique de l'Ouest: Hydrostructurale, hydrochimie et isotopie des aquifères discontinus des sillons et aires granitiques de la Haute Marahoué (Côte d'Ivoire). thèse d'Etat, Université Nationale de Côte d'Ivoire, p. 480.

Delor C, Diaby I, Yao B. 1992. Notice Explicative de la Carte Géologique à 1/200 000 ( $1^{\text {ère }}$ édition). Feuille de GrandBassam.

Faillat JP, Drogue C. 1993. Différenciation hydrochimique de nappes superposées d'altérites et de fissures en socle granitique. Hydrogical Sciences-Journaldes Sciences Hydrologiques, 38, 3(6): 215-229.

Güler C, Thyne GD, Mccray JE, Tuner AK. 2002. Evaluation of graphical and multivariate statistical methods for classification of water chemistry data. Hydrogeology Journal, 10: 455-474.

INS. 2001. Données socio-démographiques et économiques des localités, résultats définitifs par localités, région des lagunes 3, 1. Recensement Générale de la Population et de l'Habitation (RGPH) 1998.

John DD, Subbba RN, Thirupathi BR, Srinivasa KVR, Subrahmanyam A. 2007. Hydrogeochimistry of the Sarada river basin, Visakhapatnam district, Andhra Pradesh, India. Environmental Geological, (52): 1331-1342.

N'go YA., Goné DL, Savané I, Goblé MM. 2005. Potentialités en eaux souterraines des aquifères fissurés de la région d'Agboville (Sud Ouest de la Côte d'Ivoire): Caractérisation hydroclimatique et physique. Afrique Science, 1(1): 127-144.

Oga MS. 1998. Ressources en eaux souterraines dans la région du Grand 
Abidjan (Côte d'Ivoire) : Approche Hydrochimique et Isotopique. Thèse Université d'Orsay, Paris, France, p. 311.

Savané I, Soro N. 2001. Caractérisation chimique et isotopique des eaux souterraines du Nord-Ouest de la Côte : recharge des aquifères discontinus de la région d'Odienné. Africa Géoscience Review, 8(4): 379-390.

Soro N. 2002. Hydrochimie et géochimie isotopique des eaux souterraines du degré carré de Grand-Lahou et ses environs (sud-ouest de la Côte d'Ivoire). Implication hydrologique et hydrogéologique. Thèse d'Etat, Université de Cocody, p. 272.

Yidana SM, Ophori D, Banoeng-Yakubo B. 2008. A multivariate statistical analysis of surface water chemistry data. The Ankobra Basin, Ghana. Journal of Environmental Management, 86: 80-87.

Yidana SM. 2010. Groundwater classification using multivariate statistical methods: Southern Ghana. Journal of African Earth Sciences, 57: 455-469. 